\title{
Origin of magic angular momenta in few-electron quantum dots
}

\author{
W. Y. Ruan and Y. Y. Liu \\ Department of Applied Physics, South China University of Technology, Guangzhou 510641, People's Republic of China \\ C. G. Bao \\ China Center of Advanced Science and Technology (World Laboratory), P.O. Box 8730, Beijing 100080, People's Republic of China \\ and Department of Physics, Zhongshan University, Guangzhou 510275, People's Republic of China \\ Z. Q. Zhang \\ Department of Physics, Hong Kong University of Science and Technology, Clearwater Bay, Hong Kong
}

(Received 14 December 1994)

We show the effect of quantum-mechanical symmetry on determining the features of two-dimensional few-electron quantum dots, and thereby elucidate the origin of the magic numbers.

Recent advances in microfabrication have allowed the creation of quantum dots in semiconductor heterostructures by laterally confining two-dimensional electrons. ${ }^{1}$ The confining potential is, to a good approximation, parabolic and a small number $N(N=1,2,3, \ldots)$ of electrons per dot has been achieved experimentally., ${ }^{2,3}$ The electronic states of a few-electron system subjected to a strong magnetic field have been studied extensively. ${ }^{3-11}$ For example, to understand the fractional quantum Hall effect, Laughlin ${ }^{4}$ first studied the states of a three-electron system in two dimensions in a strong magnetic field and confined by a parabolic potential. Laughlin explicitly constructed the spin-polarized correlated states in the lowest Landau level and showed that they approximated the exact eigenstates well. The ground states turned out to be incompressible since only "magic numbers" of the angular momentum $L=3 k(k=1,2,3, \ldots)$ of the ground state minimize the Coulomb repulsion. Girvin and $\mathrm{Jach}^{6}$ extended the analysis to systems containing more electrons. The magic numbers were seen to exist, but the rules explaining them seemed to increase in complexity as the number of particles increased. The role of the electronelectron interaction and the effects of the external magnetic field on few-electron states in quantum dots have been studied by Maksym and Chakraborty (MC). ${ }^{7}$ By numerically diagonalizing the Hamiltonian, MC calculated the energy spectra of three- and four-electron quantum dots and pointed out that the angular momentum of the ground state of the electron systems changed with increasing magnetic field through the series of magic numbers formerly discovered by Laughlin, Girvin, and Jach. Analyses based on partial-wave mixtures did not arrive at a reasonable explanation. ${ }^{8}$ The strong efforts towards developing the general rules obeyed by the magic numbers for an arbitrary number of electrons at an arbitrary spin have not been successful because the origin of the magic numbers has not been clearly known. It is the purpose of this paper to demonstrate that the quantummechanical symmetry (Pauli principle and rotational invariance) plays a decisive role in determining the states of these few-electron systems. Consequently, the magic numbers or fractional filling factors (FFF's) in quantum Hall effects have a very simple physical origin and the rules determining the magic numbers are easy to derive algebraically.

Let us first consider a system of three electrons moving in the $x-y$ plane subjected to a parabolic confinement; the Hamiltonian reads

$$
H=\sum_{i}\left(\frac{p_{i}^{2}}{2 m^{*}}+\frac{1}{2} m^{*} \omega_{0}^{2} r_{i}^{2}\right)+\sum_{i>j} v\left(r_{i j}\right),
$$

where $\mathbf{r}_{i}$ is the position of the $i$ th particle, $v\left(r_{i j}\right)$ is the Coulomb interaction, $r_{i j}=\left|\mathbf{r}_{i}-\mathbf{r}_{j}\right|$. Introducing the center of mass (c.m.) coordinates $\mathbf{R}_{\text {c.m. }}=\left(\mathbf{r}_{1}+\mathbf{r}_{2}+\mathbf{r}_{3}\right) / 3$ and the Jacobian coordinates $\xi=\mathbf{r}_{1}-\mathbf{r}_{2}, \eta=\mathbf{r}_{3}-\left(\mathbf{r}_{1}+\mathbf{r}_{2}\right) / 2$, the Hamiltonian is then separated into

$$
H=H_{\mathrm{c} . \mathrm{m} .}+H_{I},
$$

where

$$
H_{\mathrm{c} . \mathrm{m} .}=\frac{P_{\mathrm{c} . \mathrm{m} .}^{2}}{2 M}+\frac{1}{2} M \omega_{0}^{2} R_{\mathrm{c} . \mathrm{m} .}^{2}
$$

is for the c.m. motion, and

$$
H_{I}=\frac{p_{\xi}^{2}}{2 \mu_{\xi}}+\frac{p_{\eta}^{2}}{2 \mu_{\eta}}+\sum_{i>j} u\left(r_{i j}\right)
$$

is for the internal motion, where $M=3 m^{*}, \mu_{\xi}=m^{*} / 2$, $\mu_{\eta}=2 m^{*} / 3$, and

$$
u\left(r_{i j}\right)=\frac{1}{6} m * \dot{\omega}_{0}^{2} r_{i j}^{2}+\frac{e^{2}}{4 \pi \epsilon r_{i j}},
$$

where the term proportional to $r_{i j}^{2}$ arises from the confinement. A noteworthy point is that the equivalent particleparticle potential $u\left(r_{i j}\right)$ is repulsive at small separation but attractive at large separation, with a minimum at $r_{0} \equiv\left(3 e^{2} / 4 \pi \epsilon m^{*} \omega_{0}^{2}\right)^{1 / 3}$. Therefore, the landscape of the total potential energy surface $U=\Sigma_{i>j} u\left(r_{i j}\right)$ in the multidimensional coordinate space is quite different from that without the Coulomb interaction. There is a minimum in $U$ associated with an equilateral triangle (ET) with all the side 
lengths being equal to $r_{0}$. There is also a saddle point in $U$ associated with a straight line (SL) with equal separations between adjacent particles. If the wave function is smoothly (without nodal lines) distributed around the above ET, the binding will be averagely strong and the internal motion will appear only as a gentle oscillation around the equilibrium of ET configuration. The total energy can then be minimized. Hence, the ET is the most important configuration of threeelectron systems and should be pursued by low-lying states. However, we will see that in some cases this favorable configuration is prohibited by symmetry.

For circular quantum dots, the eigenstates of $H_{I}$ are classified by the total spin $S$ and the total orbital angular momentum $L$ [denoted by $(S, L)]$. In the polarized $\left(S=\frac{3}{2}\right)$ states, the spatial part of the wave function $\Phi$ is antisymmetric; thus it is invariant under cyclic permutation $P_{(123)}$,

$$
\Phi_{L}=P_{(123)} \Phi_{L}
$$

If the electrons form an ET, a cyclic permutation is equivalent to a rotation by $120^{\circ}$. In this case, we have

$$
P_{(123)} \Phi_{L}(\mathrm{ET})=\exp \left(i \frac{2}{3} \pi L\right) \Phi_{L}(\mathrm{ET}) .
$$

Combining Eqs. (6) and (7), we obtain

$$
\left[1-\exp \left(i \frac{2}{3} \pi L\right)\right] \Phi_{L}(\mathrm{ET})=0 .
$$

In Eq. (8), if the first factor is not equal to zero, $\Phi_{L}(\mathrm{ET})$ has to be zero. This is called an ET prohibition; it occurs when $L \neq 3 k$, where $k$ is an integer. Once the ET prohibition occurs, an inherent nodal line appears in the wave functions at the ET configuration, resulting in instability. ${ }^{12,13}$ It will soon be clear that the ET prohibition is the most important factor affecting the energy spectra. In the unpolarized ( $S=$ $\frac{1}{2}$ ) states, the wave function can be expanded as

$$
\psi=\Phi_{L}^{a} \chi_{0}^{1 / 2}+\Phi_{L}^{b} \chi_{1}^{1 / 2},
$$

where $\chi_{s}^{S}$ is the spin part with the spins of particles 1 and 2 coupled to $s$, then $s$ is coupled with the spin of particle 3 to $S$. Since $\psi$ is antisymmetrized, the spatial parts are subjected to the transformation

$$
\begin{gathered}
P_{(123)} \Phi_{L}^{a}=-\frac{1}{2} \Phi_{L}^{a}+\frac{\sqrt{3}}{2} \Phi_{L}^{b}, \\
P_{(123)} \Phi_{L}^{b}=-\frac{\sqrt{3}}{2} \Phi_{L}^{a}-\frac{1}{2} \Phi_{L}^{b}
\end{gathered}
$$

under cyclic permutation. If the electrons form an ET, Eq. (7) holds also for $\Phi_{L}^{a}(\mathrm{ET})$ and $\Phi_{L}^{b}(\mathrm{ET})$. Then, instead of Eq. (8), we have

$$
\left[1+\exp \left(i \frac{2}{3} \pi L\right)+\exp \left(i \frac{4}{3} \pi L\right)\right] \Phi_{L}^{j}(\mathrm{ET})=0 \quad(j=a, b)
$$

If the first factor in Eq. (11) is not equal to zero, the ET prohibition occurs. In contrast with the $S=\frac{3}{2}$ states, now it occurs when $L=3 k$.

To see the effect of ET prohibition, the Hamiltonian was diagonalized in a model space spanned by translationalinvariant harmonic oscillator product states, ${ }^{8}$


FIG. 1. The energy spectra of a three-electron system in a quantum dot with weak confinement $\left(\hbar \omega_{0}=0.01 \mathrm{meV}\right)$. (a) $S=\frac{3}{2}$ states, (b) $S=\frac{1}{2}$ states.

$$
\hat{A}\left\{\Phi_{n_{1} l_{1}}(\xi) \Phi_{n_{2} l_{2}}(\eta) \chi_{s}^{S}\right\}
$$

where $\hat{A}$ is the antisymmetrizer. The antisymmetrization was realized by using the two-dimensional Talmi-Moshinsky transformation brackets. ${ }^{14}$ The dimension of the model space is constrained by $0 \leqslant N_{0}=2\left(n_{1}+n_{2}\right)+\left|l_{1}\right|+\left|l_{2}\right| \leqslant 24$. If $N$ is increased by 2 , the ratios of the difference in energy and the energy in the head states [the lowest one of a $(S, L)$ ] is less than $0.001 \%$. To emphasize the particle-particle correlation, a large dot $\left(\hbar \omega_{0}=0.01 \mathrm{meV}\right)$ where the Coulomb interaction is dominant has been chosen. The other parameters are taken appropriate for GaAs.

In the case of $S=\frac{3}{2}$, the spectrum is given in Fig. 1(a). It shows that the head states without ET prohibition (i.e., $L=3 k$ ) are remarkably lower. To show the internal structure, as an example, the wave function of the $\left(\frac{3}{2}, 0\right)$ head state is plotted in Fig. 2(a) as a function of $\xi$ and $\eta$ with $\theta$, the angle between $\xi$ and $\eta$, given at its optimized value $90^{\circ}$. This figure shows that the wave function is smoothly distributed 

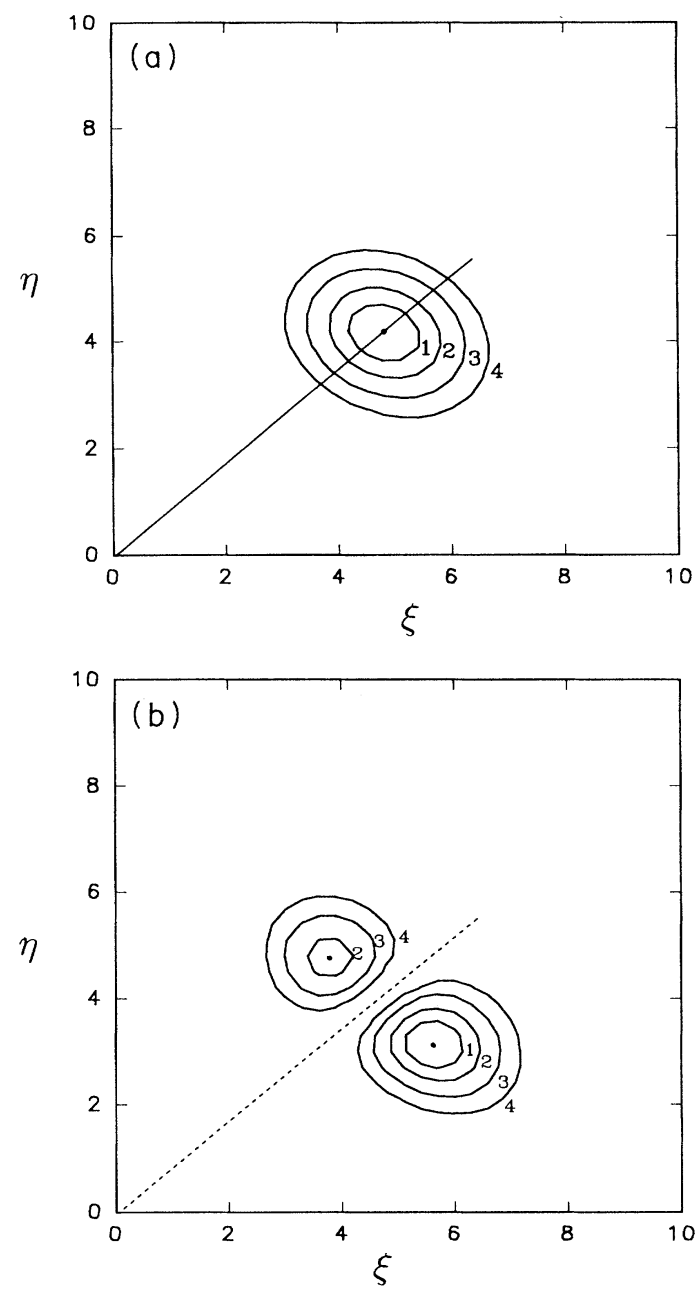

FIG. 2. $|\psi|^{2}$ plotted as a function of $\xi$ and $\eta$ with $\theta=90^{\circ}$. (a) The head state of $\left(\frac{3}{2}, 0\right)$, (b) the head state of $\left(\frac{1}{2}, 0\right)$. The contours marked by $1,2,3,4$ give $\frac{3}{4}, \frac{1}{2}, \frac{1}{4}, \frac{1}{10}$ of the maximum, respectively. The line is given by $\eta=\sqrt{3} \xi / 2$, representing the ET configuration. The length unit is $a_{0}=\sqrt{\hbar /\left(m^{*} \omega_{0}\right)}$.

around a peak on the line $\eta=\sqrt{3} \xi / 2$ at $\xi=r_{0}$. Besides, the wave function of the other $\left(\frac{3}{2}, 3 k\right)$ head states appear exactly the same as shown in Fig. 2(a). Thus in all the $\left(\frac{3}{2}, 3 k\right)$ head states, the favorable ET configuration is strongly pursued, resulting in a particularly low energy. The $L=3 k$ is just the magic numbers. Using the well-known formula $\nu=n_{e}\left(n_{e}-1\right) / 2 L,{ }^{4}$ the sequence of FFF's reads $1, \frac{1}{2}, \frac{1}{3}$, ... . From the above analysis, it is now clear that the appearance of the magic numbers is due to the ET prohibition.

In the case of $S=\frac{1}{2}$, the spectrum is given in Fig. 1(b). It shows also that head states without ET prohibition (i.e., $L \neq 3 k$ ) are remarkably lower, while the $L=3 k$ head states are remarkably higher. To show the internal structure of an ET-prohibited state, the wave function of the $\left(\frac{1}{2}, 0\right)$ head state is plotted in Fig. 2(b). The $\eta=\sqrt{3} \xi / 2$ line is now a nodal line demonstrating the ET prohibition. There are two peaks associated with a sharp and a flat isosceles triangle, respectively. The nodal line induces an energetic oscillation, and the sys- tem evolves back and forth between these two peaks by crossing the nodal line repeatedly. ${ }^{15}$

Since in the SL configuration a rotation of $180^{\circ}$ is equivalent to an exchange of the two particles sitting on the two ends, there is a SL prohibition for the $L$ even states. The SL prohibition has no effects on the low-lying states of a large quantum dot where the ET configuration is strongly pursued, but induces instability in a small dot. ${ }^{16}$ This makes the FFF's with even denominator more difficult to observe than those with odd denominator.

Our detailed observations of the wave-function distribution in the multidimensional coordinate space reveal that, if two different $(S, L)$ sequences are subjected to the same constraint from symmetry [e.g., the $\left(\frac{3}{2}, 3\right)$ and the $\left.\left(\frac{3}{2}, 9\right)\right]$, then there is a one-to-one similarity between the corresponding states of the two sequences. This fact facilitates the classification of the whole spectra. ${ }^{16}$

Experimentally, a homogeneous magnetic field perpendicular to the $x-y$ plane is applied. In the symmetric gauge, the Hamiltonian of Eq. (4) then has a new $\omega_{0} \rightarrow \sqrt{\omega_{0}^{2}+\omega_{c}^{2} / 4}$ with the cyclotron frequency $\omega_{c}=e B / m^{*}$, and has the additional Zeeman terms $\left(\omega_{c} L / 2+g^{*} \mu_{B} B_{z} S\right)$ which have no effect on the wave function but shift the energy levels. ${ }^{17}$ The Zeeman terms have preference for large angular momentum and make the unpolarized states unfavorable. As a result, the ground state will run over the series $L=3 k(k=0,1,2, \ldots)$ with increasing magnetic field. ${ }^{8}$

The above analysis is easily extended to other fewelectron systems. For example, in the case of $n_{e}=4$, the minimum of $U$ is associated with the square (SQ) configuration. However, a rotation of the SQ by $90^{\circ}$ is equivalent to a cyclic permutation $P_{(1234)}$ (an odd permutation). Thus in the polarized $(S=2)$ states (where there is only one component for the spatial part), instead of Eqs. (8) and (11), we have

$$
\left[1+\exp \left(i \frac{\pi}{2} L\right)\right] \Phi(\mathrm{SQ})=0
$$

The magic numbers are those values satisfying $[1+\exp (i \pi L / 2)]=0$ (otherwise, the SQ-prohibition occurs); they are $L=2(2 k+1)$. Consequently the FFF's read $1, \frac{3}{5}, \frac{3}{7}, \frac{1}{3}, \ldots$.

In the case of $n_{e}=5$, there are two minima in $U$ having similar energies. One is associated with the equilateral pentagon (EP) and the other one is associated with the centered square (CSQ, i.e., a square with an extra particle at the center). A rotation of the EP by $72^{\circ}$ is equivalent to a cyclic permutation $P_{(12345)}$ (an even permutation), a rotation of the CSQ by $90^{\circ}$ is equivalent to a cyclic permutation $P_{(1234)}$ as before. Thus, in the polarized $\left(S=\frac{5}{2}\right)$ states, instead of one, we have two equations as

$$
\begin{gathered}
{\left[1-\exp \left(i \frac{2}{5} \pi L\right)\right] \Phi(\mathrm{EP})=0} \\
{\left[1+\exp \left(i \frac{\pi}{2} L\right)\right] \Phi(\mathrm{CSQ})=0 .}
\end{gathered}
$$


The magic numbers are those values of $L$ giving $\left[1-\exp \left(i \frac{2}{5} \pi L\right)\right]$ or $[1+\exp (i \pi L / 2)]$ to be zero; they are $0,2,5,6,10,14,15,18,20,22, \ldots$. Accordingly, the FFF's read $1, \frac{5}{7}, \frac{2}{3}, \frac{5}{9}, \frac{1}{2}, \frac{5}{11}, \frac{2}{5}, \frac{5}{13}, \frac{1}{3}, \ldots$.

The above derived magic numbers are exactly the same as those found by exactly diagonalizing the Hamiltonian. ${ }^{4-9,16}$ Now, the origin of magic numbers is clear. It is noticeable that the filling factor $\frac{1}{3}$ is common in a number of fewelectron systems (at least, it is common in $n_{e}=3,4,5$, and 6 systems). This explains why the signal corresponding to $\frac{1}{3}$ is particularly strong in experiments.

In the above procedure of analysis, parabolic confinement has been assumed and the c.m. motion is separable from the relative motion. In the case of nonparabolic confinement, there is hybridization of c.m. and relative motion; however, the magic numbers remain the same. ${ }^{16}$ Thus the magic num- bers are insensitive to details of the dynamics; the dominant factor is the constraints of symmetries.

The method presented here is applicable to other strongly correlated few-body systems such as the multiexcited states of atoms and nuclei where the shell model is no longer valid. ${ }^{18}$ The outcome is that it projects the spectrum into rotation bands and the order of bands can be well understood. Traditionally, our physical understanding of constraints arising from symmetries (e.g., Pauli principle) relies seriously on the independent particle model. The present method provides a key to understanding the symmetry constraints in strongly correlated systems where the concept of a single-particle state is no longer valid.

This work was supported by the National Natural Science Foundation of China, Grant No. 19134020, the Foundation of the National Educational Committee, and the Guangdong Natural Science Foundation.
${ }^{1}$ T. Chakraborty, Comments Condens. Matter Phys. 16, 35 (1992).

${ }^{2}$ B. Meurer, D. Heitman, and K. Ploog, Phys. Rev. Lett. 68, 1371 (1992).

${ }^{3}$ R. C. Ashoori, H. L. Stormer, J. S. Weiner, L. N. Pfeiffer, K. W. Baldwin, and K. W. West, Phys. Rev. Lett. 71, 613 (1993).

${ }^{4}$ R. B. Laughlin, Phys. Rev. B 27, 3383 (1983).

${ }^{5}$ R. B. Laughlin, Phys. Rev. Lett. 50, 1395 (1983).

${ }^{6}$ S. M. Girvin and Terrence Jach, Phys. Rev. B 28, 4506 (1983).

${ }^{7}$ P. A. Maksym and Tapash Chakraborty, Phys. Rev. Lett. 65, 108 (1990).

${ }^{8}$ P. A. Maksym and Tapash Chakraborty, Phys. Rev. B 45, 1947 (1992).

${ }^{9}$ Pawel Hawrylak and Daniela Phannkuche, Phys. Rev. Lett. 70, 485 (1993).
${ }^{10}$ S-R. Eric Yang, A. H. Macdonald, and M. D. Johnson, Phys. Rev. Lett. 71, 3194 (1993).

${ }^{11}$ J. J. Palacios et al., Phys. Rev. B 50, 5760 (1994).

${ }^{12}$ C. G. Bao, Z. Phys. D 22, 557 (1992).

${ }^{13}$ C. G. Bao, Few-Body Systems 13, 41 (1992).

${ }^{14}$ W. Y. Ruan and Y. Y. Liu, Commun. Theor. Phys. (to be published).

${ }^{15}$ Static state in quantum mechanics corresponds to cyclic motion in the classical mechanics. For references, see C. G. Bao, Phys. Rev. A 47, 1752 (1993).

${ }^{16}$ W. Y. Ruan et al. (unpublished).

${ }^{17}$ N. F. Johnson and M. C. Payne, Phys. Rev. Lett. 67, 1157 (1991).

${ }^{18}$ C. G. Bao et al. (unpublished). 